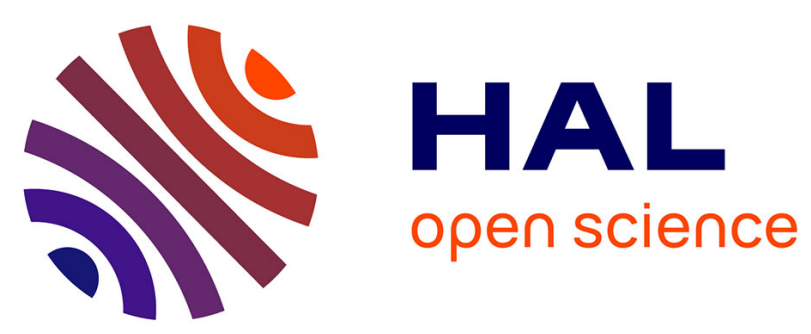

\title{
An array of physical sensors and an adaptive regression strategy for emotion recognition in a noisy scenario
}

Francesco Mosciano, Arianna Mencattini, Fabien Ringeval, Björn Schuller, Eugenio Martinelli, Corrado Di Natale

\section{- To cite this version:}

Francesco Mosciano, Arianna Mencattini, Fabien Ringeval, Björn Schuller, Eugenio Martinelli, et al.. An array of physical sensors and an adaptive regression strategy for emotion recognition in a noisy scenario. Sensors and Actuators A: Physical , 2017, 267, pp.48-59. hal-01993393

\section{HAL Id: hal-01993393 \\ https://hal.science/hal-01993393}

Submitted on 24 Jan 2019

HAL is a multi-disciplinary open access archive for the deposit and dissemination of scientific research documents, whether they are published or not. The documents may come from teaching and research institutions in France or abroad, or from public or private research centers.
L'archive ouverte pluridisciplinaire HAL, est destinée au dépôt et à la diffusion de documents scientifiques de niveau recherche, publiés ou non, émanant des établissements d'enseignement et de recherche français ou étrangers, des laboratoires publics ou privés. 
MANUSCRIPT

5

Authors: Francesco Mosciano ${ }^{1}$, Arianna Mencattini ${ }^{1}$, Fabien Ringeval ${ }^{2}$, Björn Schuller ${ }^{3,4}$, Eugenio Martinelli ${ }^{1}$, Corrado Di Natale ${ }^{1}$

\author{
1. Dept. of Electronic Engineering, University of Rome Tor Vergata, Rome, Italy \\ 2. Laboratoire d'Informatique de Grenoble, Université Grenoble Alpes, Grenoble, France \\ 3. Chair of Complex \& Intelligent Systems, University of Passau, Passau, Germany \\ 4. Department of Computing, Imperial College London, London, UK.
}

Contacting author:

Prof. Eugenio Martinelli

Department of Electronic Engineering, University of Rome Tor Vergata

Via del Politecnico 1; 00133 Roma

Phone: +390672597259

Fax: +39062020519

Email: martinelli@ing.uniroma2.it recognition in a noisy scenario 
Abstract

Several studies demonstrate that since emotions are spontaneously manifested through different measurable quantities (e.g. vocal and facial expressions), this makes possible a sort of automatic estimation of emotion from objective measurements. However, the reliability of such estimations is strongly influenced by the availability of the different sensor modalities used to monitor the affective status of a subject, and furthermore the extraction of objective parameters is sometime thwarted in a noisy and disturbed environment. This paper introduces a personalized emotion estimation based on a heterogeneous array of physical sensors for the measurement of vocal, facial, and physiological (electro-cardiogram and electrodermal) activities. As a proof of concept, changes in the levels of both emotion reactiveness and pleasantness are estimated under critical operative conditions. The estimator model takes advantage from the timevarying selection of the most relevant non-spurious sensors features and the adaptation of the k-nearest neighbour paradigm to the continuous identification of the most affine model templates. The model, once trained, demonstrated to autonomously embed new sensorial input and adapt to unwanted/unpredicted sensor noise or emotion alteration. The proposed approach has been successfully tested on the RECOLA database, a multi-sensorial corpus of spontaneous emotional interactions in French.

Keywords: Sensor Array; Adaptive regression strategy; Emotion recognition; 
Several studies show that quantitative measurements of human expression can be used to estimate psychological and physical conditions in humans [1,2,3]. Artificial empathic systems are expected to be of benefit in many diverse domains such a precision medicine, personalized care and therapy, customer satisfaction studies, or web profiling, to mention a few [4-6]. According to a commonly accepted model [7], the complexity of emotions may be simplified using two features describing the level of reactiveness to stimuli, named arousal, and the level of attractiveness/averseness of an emotion, named valence.

A subject may manifest his/her affective condition using facial mimic, voice alteration, electro-cardiogram deviation from normal status and electro-dermal activities modification due for example to sweating. The increasing interest in modalities that complement to audio/visual is motivated by the growing availability of wearable devices that include physiological sensors, such as electro-cardiogram and electro-dermal activity, at an affordable cost. We indicate such plethora of verbal/nonverbal messages as a multi-sensorial representation of the emotion. Previous studies [8-11] indicate that there can be a strong correlation between alterations in sensor acquisition and in the level of arousal and valence observed over small time intervals of about half a second. If correctly estimated, such relevance can be exploited in order to construct a reliable model able to predict the affective status in the future. The most important implication can be to optimize the therapeutic plans, advertising strategies, or not less fascinating, a cybernetic retelling of the home-sweet-home paradigm. With respect to subject independent emotion estimation, customized emotion prediction adds the simplification of not requiring a large amount of labelled data for training the model and a greatest robustness to an affective content baseline. In fact, personalized estimation can be achieved without the need to normalize the descriptors but rather exploiting data variability within the session in favour of increased prediction performance. At the same time, it may suffer from reduced information about past affective content that can negatively impact on the capability to accurately anticipate the subject's affective status and consequently design prompt actions. Unfortunately, the complexity of the scenario to model, and the diverse sources of variability present, make it very difficult to develop a robust emotion prediction system even in case of customised affective estimation, as graphically illustrated in Fig. 1, where the inter-relations between problems and sources of variability are outlined.

In analogy to what happens in very complex sensor systems, this scenario poses many problems originally solved in different engineering fields. Hence, in the following sections, we present and discuss the main sources of variability that should be accounted for in multi-sensorial emotion prediction, providing, at the same time, the original contextualization in sensor networks management. As outlined in [12], no sensor fusion technique is superior to others in all contexts, but there can be an optimal configuration for a specific application. With this in mind, in this work, we intend to implement the C3 paradigm of multi-sensor fusion theory [13], i.e., complementary, competitive, and cooperative. A complementary configuration allows the sensors to not directly depend on each other, but to be combinable in order to give a more complete image of the phenomenon under observation. Different sensors may acquire different cues of the same affective condition. In a competitive architecture, each sensor delivers independent measurements of the same property. Examples of competitive sensors are redundant configuration and fault tolerance that allow the possibility to capture the same general affective condition using a different modality (e.g., negative valence, positive arousal, etc.). A cooperative sensor network uses the information provided by each sensor modality to increase the degree of knowledge of a given property, overcoming individual inaccuracy and uncertainties. To demonstrate the effectiveness of the cooperative fusion approach, we will compare performance of individual sensor modalities in emotion recognition with those obtained by a multi-sensor configuration. 
The success of such an architecture lies in the hybrid fusion of characteristics that, if properly accounted, ensure robustness, reliability, and effectiveness to the solution. In emotion prediction, complementary requires that different aspects of the same affective condition are measured, and this is achieved through the different sensor modalities. Competitiveness asks for the independence of the different sensor acquisitions, obtained by different hardware, physical principles, as well as acquisition settings. Finally, cooperativeness allows the information acquired from different sensors to be synergistically aggregated to construct a unified model of prediction, as will be demonstrated by a dynamic adaptive procedure. The novelty of our approach lies in the strategy used to implement the C3 paradigm, and in how it is applied to personalized emotion prediction. First of all, each modality of the affective manifestation is acquired through a dedicated sensor device, later indicated as AUDIO, VIDEO, ECG, and EDA. The four sensors acquire a onedimensional audio speech signal, the video sequence of the upper body of the subject, the ECG signal, and the EDA signal. Sensor spurious data (blue subtree node in Fig. 1) may occasionally or permanently occur due to ill positioning of the sensors, subject movement, power blackout, or transmission interruption, for example. Such situations may cause a modality unavailable or unreliable, and if this issue is not properly accounted for, it may dramatically alter the prediction effectiveness [14-16]. Second, when all the sensor modalities are correctly acquired and all sensors work properly, some specific descriptors extracted from a single modality may result unreliable or missing (green subtree node in Fig. 1). This may be due to unexpected values of the subject's physiological state, or to the spectral content of the speech, or to particular conditions of video acquisition that cause incorrect working of the face landmarking procedure and produce spurious feature values. Such a situation, sometimes addressed using sensor redundancy $[17,18]$ or algorithmic corrections [19], such as cooperative integration strategies [15], may alter the goodness of prediction and bring the module to produce out-of-range predictions.

When multiple sensors are embedded into a unique device with the aim to estimate the affective status of the subject, the descriptors extracted can be highly heterogeneous either in distribution over the training population or in the range and average values (headed by the pink subtree node in Fig.1). Sensor fusion techniques then allow to embed in a unique sensorial system all the information related to the subjective externalization of emotions using cooperative strategies able to combine descriptors with different range and relevance levels $[1,20]$.

One of the most crucial aspect is whether or not leaving the possibility to the model to integrate the new data along with the corresponding prediction in the training knowledge-base for re-calibration of the entire sensor networks (yellow subtree node in Fig.1). Abandoning the old paradigm of a static configuration, even if it may assure a low computation effort, is a delicate aspect to consider. Reliability of the novel acquired predictions should be accurately accounted for to avoid the risk of confounding the retrained model and producing erroneous future predictions and instability of the dynamically configured system [8].

In order to account for all of the outlined problems, in this work, we propose a novel dynamic prediction approach. First, the configuration allows to face descriptors' unreliability, and data missing occurring in new tested instances. Correlation based feature selection methodologies are applied to all the descriptors in the training thus equating the relevance of each modality by the objective correlation metric and selecting the optimal descriptors to build the model. At each prediction, a new model is re-trained by selecting the templates from the training set that are in the nearest neighbourhood of the data in test. Finally, the new data along with the respective output predictions are included in the training set when the output level is comparable with those already acquired to avoid a training confounding effect. 
The remainder of this article is organized as follows. In Section 2, we will describe the dataset used for the experiments and provide a sketch of the descriptors extracted. Then, in Section 3, we will describe the diverse modules composing the system and illustrate the way they cooperate. Next, in Section 4, we will detail out the experiments run, and in Section 5, we will provide some hints for discussion. Finally, in Section 6, we will draw conclusions.

\section{Physical multimodal sensors and data recording}

In order to provide experimental results on the novel method proposed for the personalized emotion estimation using multi-sensorial acquisition, we consider as benchmarking database the RECOLA (REmote COLlaborative and Affective interaction) corpus [20], a recently developed multimodal corpus of spontaneous interactions in French. It has been used as benchmarking dataset for the multimodal affect recognition sub-challenge in the last two editions of the Audio/Visual Emotion recognition Challenge (AVEC'15, AVEC'16) $[9,10]$. The RECOLA corpus, freely available at https://diuf.unifr.ch/diva/recola/, was recorded to study socio-affective behaviours from multimodal data in the context of remote collaborative work, for the development of computer-mediated communication tools. Spontaneous and naturalistic interactions were collected during the course of a collaborative task that was performed remotely in dyads through video conference. Physical multimodal sensor acquisition, i.e., audio (AUDIO), video (VIDEO), electro-cardiogram (ECG) and electro-dermal activity (EDA), were simultaneously recorded from 27 Frenchspeaking subjects. Audio data were captured by unidirectional headset microphones (AKG C52OL) and recorded using the Audacity software at $44.1 \mathrm{kHz}$ with 16 bits. An external sound card (Lexicon Omega) was used to split the audio data in order to be simultaneously processed by Skype and Audacity. As this solution was not applicable to the video sensors without compromising the frame rate, two HD webcams (Logitech C270) were used for each participant. The first webcam only captured the video data to be used for the Skype video-conference, whereas the second webcam was used to record both audio, from the built-in omnidirectional microphone, and video with the software provided by the manufacturer; audio was recorded at $48 \mathrm{kHz}, 16$ bits, and brightness auto adjustments were turned off for video recording. Regarding physiological data, the Biopac MP36 unit and the Biopac Student Lab software were used to record both EDA and ECG signals with a sampling frequency of $1 \mathrm{kHz}$.

Even though all subjects speak French fluently, they have different nationalities (i.e., French, Italian or German, cf. below), which thus provides some diversity in the encoding of affect especially in emotion externalization through speech and facial mimic. Out of the 27 speakers, only 18 gave their consent for publication and sharing their data. In order to train and validate a prediction model, time-continuous ratings ( $40 \mathrm{~ms}$ binned frames) of emotional arousal and valence were also acquired by six gender balanced (three women and three men) French-speaking assistants. Since participants showed emotions mostly at the beginning of their interaction, the annotations were collected during the first five minutes of all recordings. In order to collect a unified gold standard from the six annotations available for each speaker, a normalization technique based on the Evaluator Weighted Estimator introduced in [22], that demonstrated a significant improvement ( $p<0.001$ for correlation coefficient using Student t-test) in the inter-rater reliability for both arousal and valence, was applied. For the task of demonstrating the effectiveness of personalized emotion estimation in real-life condition, and according to previous studies [23,31], we reduced the sampling rate of the signal acquired to $400 \mathrm{~ms}$, leading to sequences with a duration of 750 samples.

Eligibility criteria. Personalized emotion estimation requires an initial phase of acquisition with provided annotations in both the arousal and valence dimensions, then followed by a session of prediction. To do this, at most an initial $30 \%$ of the sequence should be used for training the model and the remaining $70 \%$ part for testing. In light of this, we eliminated conversations in which emotions abruptly changed after about the first 
third of the sequence. Such sequences would cause the inability to achieve reliable performance in testing, since the model would have been tested on affective contents far different from the ones in training, occurring in an unrealizable task of predicting the unknowable instead of the unknown. At the same time, we selected subjects balancing for gender. The present method will be then verified on the conversations of 10 speakers whose metadata are reported in Table 1. Out of the speakers, seven ones are French, two ones are Italian and only one is German. The average age is 21.4 years ( $\sigma=2.5$ years). Five speakers are female and five ones are male. In addition, the fifth and sixth columns in Table 1 list the average and the standard deviation values of arousal and valence for each speaker. Data Descriptors. Each of the four (AUDIO, VIDEO, ECG, and EDA) provided a signal that was further processed using well-founded feature extractors: for the acoustic features, we used the well standardised and broadly used ComParE set of low-level-descriptors, which includes 65 acoustic descriptors with their first order derivate (130 acoustic descriptors) extracted through the openSMILE (Release 2.0) [24] open source software. For the facial action features, 49 landmarks of the face are returned using the Supervised Descent Method (SDM) [25] applied to each frame. According to the Facial Action Coding System (FACS) [26], human facial movements may be taxonomized by their appearance on the face. Firstly, developed by the Swedish anatomist Carl-Herman Hjortsjö, this standard was later adopted by P. Ekman and W.V. Friesen, and published in 1978 [26]. Movements of individual facial muscles are encoded by FACS from slightly different instant changes in facial appearance. Based on the Action Units (AUs) of the subject, which quantify the activity of groups of facial muscles according to the FACS lexicon, it is common to systematically categorize the facial expression of emotions, and it has proven useful to psychologists and to animators. The AUs of the subject were detected starting from high level processing applied to the 49 face landmarks $[27,28]$. Post processing performed on the AUs led to a set of 40 visual descriptors: numerical details can be found in [29]. Regarding electro-cardiogram descriptors, 28 spectral features are extracted from the ECG signal along with their first order derivatives additionally computed on all features except for two (heart rate and heart rate variability) providing 54 features in total; finally, concerning the EDA signal that reflects a rapid, transient response called skin conductance response (SCR), and a slower, basal drift called skin conductance level (SCL) [21,30,31], 30 features are computed along with the first order derivative for all, providing 60 features in total. The four modalities collected led to a total of 284 descriptors for each subject. Beyond the largely proven relevance of audio/visual descriptors for emotion estimation, also physiological signals have demonstrated to correlate with emotion $[32,33]$ - in particular to arousal, despite not being directly perceptible as the way audio-visual data are to humans. Despite controversies that arose about the relation between peripheral physiology and emotions [34,35], autonomic measures have the strong advantage to be easily and continuously monitored using wearable sensors [3638]. To verify such controversial relevance in our context, Fig. 2 illustrates the average absolute values of the Pearson's Correlation Coefficient (CC) computed over each modality with respect to the arousal (left) and valence (right) dimensions for the 10 speakers. Noteworthy is the fact that there is a large variability over the 10 subjects of how each modality impacts on the expected output, thus giving sense to the personalized strategy and to the need of implementing a dynamic selection of the descriptors in the prediction. Moreover, it can be noted that if the AUDIO modality is dominantly correlated with the arousal dimension (see for example speakers P28 and P56), in case of valence all the four modalities balance their importance and support the need for a multimodal strategy.

\section{Dynamic Feature selection and regression strategy}


In this section, we will describe the innovative architecture presented for the task of multi-sensorial emotion recognition. The proposed sensor network consists of the five distinct cooperating modules represented in Fig. 3 each dealing with a specific predictive property. Interactions of the modules are also indicated.

1. The Missing Measure Protection (MMP) module aims to protect against missing or unreliable feature values that can occur in one of the sensors. As a frequent condition, a subset of features acquired by a single sensor may be missing due to the diverse situations depicted by the scheme in Fig. 1 . When this situation occurs, the system dynamically switches off features that are missing or unreliable during model construction and prediction. More specifically, the MMP module checks whether features acquired by a single sensor modality are out of range with respect to the corresponding training reference values or totally missing. By indicating with $x_{i}$ the $i$-th feature in a test sample, and with $y_{i 1}, \ldots, y_{i n}$ the corresponding $n$ values in the training set, then if $\left|x_{i}-\mu_{i}\right|>6 \sigma_{i}$ (condition 1 ), where

$$
\begin{aligned}
& \mu_{i}=\frac{1}{n} \sum_{j=1}^{n} y_{i j}, \\
& \sigma_{i}^{2}=\frac{1}{n-1} \sum_{j=1}^{n}\left(y_{i j}-\mu_{i}\right)^{2},
\end{aligned}
$$

Feature $y_{i}$ is totally eliminated from the model computation for the prediction of that test sample.

The MMP module represents an unsupervised processing block required to maintain sufficiently high accuracy also in accidental situations such as a power blackout, or a general inability to acquire data (e.g., a temporary subject out of focus event). MMP may have the consequence to reduce the number of features used in the construction of the regression model. The MMP module provides the test input to the regression model (RM).

2. The Dynamic Training Template Recruitment (DTTR) module aims to select, for each data in test, the nearest measured values in the training set for the construction of the optimal prediction model. The DTTR module is based on the well-known concept of training templates. When a test data is processed, the Euclidean distance between its feature vector and those of the training data are computed, after min-max normalization. The smallest $\mathrm{K}$ distances are identified and the corresponding $\mathrm{K}$ training samples are taken as model templates for further model construction. The module DTTR is an unsupervised block that mimics the K-nearest neighbours (K-NN) pattern recognition paradigm. It consists in identifying and extracting the $\mathrm{K}$ nearest $\mathrm{K}$ templates in training that exhibit the highest affinity with the data in test (provided by the MMP module) in terms of a given distance metric. Here, $\mathrm{K}$ is a fixed parameter selected with the requirement to store at most $\mathrm{K}$ elements at each time provided that the starting training set contains 200 samples that enlarges as time goes on. In our work, we selected $\mathrm{K}=200$ as a trade off between computation burden and template representativeness.

3. The Maximum Relevance Modality Selection (MRMS) module aims to select in the training set the modalities that best correlate with the expected output to build the predictive model. The MRMS method selects features that mostly correlate with the output level of arousal and valence independently. Denoting with $y_{i}$ the $i$-th feature in the training set, $i=1, \ldots, Q$, being $Q$ the total number of features provided by the DTTR module, and with $z$ being the expected level of the output property at a given time (provided by the expert evaluation), then the MRMS criterion finds a subset of $D$ features $\tilde{y}_{i}, i=1, \ldots, D \leq Q$, to maximize the following quantity 
where $I\left(y_{i}, z\right)$ represents the mutual information between feature $y_{i}$ and $z$ defined as

$$
I\left(y_{i}, z\right)=\sum_{y_{i}} \sum_{y_{k}} p\left(y_{i}, z\right) \log \left(\frac{p\left(y_{i}, z\right)}{p\left(y_{i}\right) p(z)}\right)
$$

where $p\left(y_{i}, z\right)$ is the discrete joint probability distribution (or probability mass function) of variables $y_{i}$ and output $z$, and $p\left(y_{i}\right)$ represents the marginal probability mass function of the variable $y_{i}$. The mutual information between two variables tells us how much one of the two variables can be statistically explained by the other, hence, in our case, to which extent the output can be explained by a feature. Note that, the term in eq. (3) maximizes the mutual information between each feature and the expected output, and it is also called Relevance Term. Further details can be found in $[1,40]$. Feature selection is performed modality by modality and therefore we named it as maximum relevance modality selection. As outlined in the Introduction, feature redundancy is not solved at this step as a strategy to increase system robustness. However, to assure the system works even in presence of highly correlated features, we decided to implement a regression module based on partial least square regression. It is well known that this kind of technique solves collinearity problem since the input space is preliminary projected into a smaller domain with the immediate effect of reducing such phenomenon.

4. The Regression Module (RM) aims at predicting the level of arousal and valence of the new data (test input from the MMP module). It is built using the template (DTTR) and the features selected in the training set (MRMS). The Partial Least Square (PLS) regression approach is applied to arousal and valence prediction, iteratively and adaptively trained according to the selected and reduced set of features from the training set $[42,43]$. In our work, we built a separate PLS model for arousal and valence expected output. The optimal number of latent variables for each regression model is chosen as the value for which the root mean square error of the validation (RMSECV) computed in the validation dataset (20 splits, 10 iterations, and mean centring in both input and output variables) reaches the minimum value [45].45 The corresponding test output values are sent to the dynamic measure integration (DMI) module.

The MRMS and RM modules constitute the supervised prediction blocks that use the information about the expected levels of arousal and valence in the training set to construct the optimal predictive model. PLS regression has been preferred over other available regression approaches as it accounts for data collinearity. Feature redundancy, related to the competitive property of sensor fusion in C3 paradigm, is a crucial concept here with positive effects. In fact, fault tolerance can be supported also by a certain level of redundancy that may protect from the accidental fault of a single measurement in a given sensor modality. For this reason, methods that preliminarily reduce redundancy (such as stepwise or sequential floating forward feature selection), or regression approaches that generally suffer from excessive feature redundancy (such as ANN) may not have a chance of success in respect of robustness.

5. The Dynamic Measures Integration (DMI) module aims to selectively enlarge the training set with the predicted data (test output) to increase the sensor network knowledge-base and improve its capability to deal with future unknown affective conditions. This semi-supervised module provides a feedback input to the approach (to the DTTR and the MRMS modules) by dynamically increasing the training set with the selective inclusion of the predicted test data, aiming to maintain a high accuracy 
also if unknown affective conditions are presented as input to the system, especially when the training set did not embrace all the plethora of possible emotions. Of course, to simultaneously maintain the robustness of the system to prediction errors, only data whose level of predicted emotions (arousal and valence separately) is within a certain range with respect to the already predicted content, are included. In particular, indicating with $\sigma_{z}$ and $\mu_{z}$ the standard deviation and the mean values of the predicted output $z$ computed up to time $t$, then the new predicted value $\hat{z}$ at time $t+1$ will be added to the training labels only if

$$
\left|\hat{z}-\mu_{z}\right|<3 \sigma_{z}
$$

Otherwise, the sample is not included in the knowledge-base (here, three times the standard deviation has been considered as a reliable range). The DTTR module will then consider the additional data for selecting the K-NN templates in case of high affinity with the new test data. The selected constrain is needed to avoid the system has to predict output values out of the range (i.e., the need to extrapolate data). For this reason, the training set has to be selected in order to cover a wide range of output values for arousal and valence. In practical applications, it means that it is impossible to ask the system to predict strongly positive valence values if it has been trained only on negative and low positive values.

\section{Experiments}

In order to demonstrate the effectiveness of the proposed strategy, we performed the following extensive experiments.

First, in Test 1, the dynamic approach was applied considering all the sensor modalities in a whole and compared with a static configuration (sensor fusion at feature level). Such an experiment allows us to predict the potential improvement of the proposed novel configuration. The prediction capability was assessed through the Concordance Correlation Coefficient (CCC) [44], which combines the Pearson's correlation coefficient (CC) with the square difference between the mean of the two compared time series as follows:

$$
\operatorname{CCC}(x, y)=\frac{2 \rho \sigma_{x} \sigma_{y}}{\sigma_{x}^{2}+\sigma_{y}^{2}+\left(\mu_{x}-\mu_{y}\right)^{2}}
$$

where $\rho$ is the Pearson correlation coefficient between two time series (e. g., prediction and gold standard) $x$ and $y, \sigma_{x}{ }^{2}, \sigma_{y}{ }^{2}, \mu_{x}$ and $\mu_{y}$ are the variances and the mean values of the two time series. Such metric, used as official scoring metric for the AVEC'15 and AVEC'16 challenges, outperforms other kind of standard performance measures (e.g., CC or RMSE) on this task in terms of suitability and meaningfulness, since predictions that are well correlated with the gold standard but shifted in value are penalised in proportion to the deviation while predictions that averagely approach each other but do not correlate, still exhibit low CCC values with respect for example to RMSE [45].

Second, in Test 2, we evaluated the robustness of the proposed system to noisy or unreliable feature values, assuming that also under a sensor operation status, some of the measured values cannot be used for the prediction, as highlighted in Fig.1. Single feature unreliability was artificially injected by randomly setting the 
$10 \%$ of the descriptors of each modality in test in turn to spurious or missing values for the entire duration, and applying the multimodal prediction approaches (dynamic and static configurations) as in Test 1.

All the experiments were conducted on the 10 subjects selected. Each feature sequence was originally reduced at the beginning and at the end of an amount of samples needed to eliminate N/A descriptors in the video modality probably arisen from excessive movements of the subject at the beginning and at end of the task. The resulting sequences included 671 samples and had a duration of $268.4 \mathrm{~s}$. Then, for each subject, we considered a training set represented by the initial 200 samples of each modality with the corresponding annotations ( $80 \mathrm{~s}$ ), and the remaining 471 samples (188.4 s) for testing.

\section{Results}

In this section, we provide numerical results of the two tests performed.

Test 1. The dynamic approach was applied considering all the sensor modalities in a whole and compared with a static configuration, i.e., a PLS regression module trained on the same original training set of the dynamic configuration with the addition of the MRMS feature selection module. Test 1 demonstrated that, the multimodal configuration allows to obtain a more reliable emotion prediction, being more robust to the intra-subject variability occurring during the predictions of the emotion thanks to a higher level of adaptation. Median (std) CCC values of $0.62(0.12)$ and $0.60(0.20)$ for the arousal (dynamic and static configuration) and of $0.30(0.26)$ and $0.05(0.25)$ for the valence (dynamic and static), respectively, have been obtained. In addition, Fig. 4 illustrates the boxplots of the CCC values obtained. A paired t-test was run to estimate the statistical significance of the achieved improvement. It can be noted that, the best improvement was obtained in the valence estimation which is the most difficult affective dimension to estimate according to the literature $[1,2,3,9,10,24,45]$. In Fig. 5, we also showed the result of the arousal (top) and valence (bottom) prediction obtained using the dynamic configuration for the speaker P16. The blue line identifies the prediction achieved, the red line represents the expected affective dimension, and the green squares locate the training session. The corresponding CCC values obtained are also indicated.

In order to compare the performance of the multimodal configuration with those of each single modality, in Table 2 we listed the median CCC values along with the corresponding standard deviation values of the single modalities. Note that the multimodal dynamic configuration outperforms each of the single modalities using the same dynamic configuration in case of arousal prediction, and strongly improves the results in case of valence prediction.

Test 2. Single feature unreliability has been simulated by randomly setting $10 \%$ of the descriptors of each modality to spurious or missing values for the entire duration and applying the multimodal prediction approaches (dynamic and static configurations) as before in Test 1. Test 2 aims at proving the effectiveness of the proposed approach to protect the system against random (occasional) descriptor unreliability due, for example, to errors in the post-processing, in the storage, or in the transmission of data. Of course, if correlated features are simultaneously missing and those features are all significant for the prediction, the system performance can heavily degrade. On the other hand, we injected missing data only on the $10 \%$ of descriptors for each modality hence avoiding, on average, to fall in such critical scenario. 
Figure 6 shows the boxplots of the CCC values of the proposed strategy for arousal (upper) and valence (lower) prediction. The proposed dynamic multimodal configuration (Dyn Mul) is compared against the static multimodal configuration (Stat Mult) as in Test 1.

From the observation of the results, it is evident that the static configuration is critically influenced by spurious values injected in the $10 \%$ of the feature values of a single modality. This is due to the fact that the static configuration selects the optimal features only once at the beginning of the procedure. Hence, if a descriptor is selected and then it assumes spurious values during test, all the predicted output sequence results in failure. On the contrary, the proposed approach, thanks to the dynamic selection of the features and the outlier elimination procedure forwarded by the data acquired in test, eliminates the features that exhibit spurious values in the test and protects the prediction from failures.

\section{Discussion}

In this study, we presented a novel multi-sensor platform and fault-tolerance processing strategies able to integrate multimodal emotion data collection, and robustness to occasional data unreliability during test. Moreover, the system is able to incorporate the new input data along with the corresponding estimated affective content, thanks to a dedicated architecture able to protect the system from drift and instability of the future predictions. The proposed architecture satisfies the C3 paradigm being complementary, competitive, and cooperative as demonstrated in the Tests 1 and 2.

An interesting part of this study was to investigate the distribution over the four sensor modalities of the features selected at each frame for the prediction of arousal and valence. Figure 7 shows a stacked bar graph in which for each speaker the length of each coloured bar represents the relative frequency of selection of the modality. Modalities are identified by colours. Noteworthy is the fact that, if for arousal the audio modality is almost always the most selected one, for the valence dimension the frequency of selection is much more distributed over the modalities. This fact demonstrates once more the usefulness of the multimodal approach to account for the inter-subject variability. Moreover, the diverse distribution over the speakers in the valence prediction allows to assign equal importance to the four modalities and in general reduces the superiority of the audio modality that is manifested instead in the arousal prediction.

One of the most interesting properties of the system presented here is the ability to cope with the unexpected lack of data (an entire sensor modality or a subset of feature values). To further demonstrate this ability, we ran a dedicated experiment with the aim to simulate a progressive, not instant, sensor missing. We artificially set to missing values an increasing percentage of feature values for the same modality (video in the experiment), until one obtains the entire fault of that sensor modality. The simulated scenario is illustrated in Fig. 8 for the valence dimension. The progressive degradation of sensor performance is graphically illustrated by the insets shown over the prediction at three time instants ( $80 \mathrm{~s}, 160 \mathrm{~s}$, and $268 \mathrm{~s}$ ). Coloured bars represent the four modalities as indicated in the legend. The bottom bars represent the percentage of non-spurious features for each modality and shows that features in the video modality progressively move entirely towards fault. The top bars indicate the corresponding percentage of features selected in each modality. Note that, a subset of features from the video modality is still chosen by the algorithm at the beginning of the prediction, but the percentage of selection goes down to zero as long as the percentage of spurious video features increases (268 s). 
As a final evidence of the effectiveness of the dynamic multimodal configuration proposed in this work, we staged a more critical scenario, in which an entire modality in turn is missed by assuming that one of the four input signals is not available during the entire duration of test. The comparative approaches are again the dynamic and the static multimodal architectures. A total of four different simulation results are collected, one for each missing modality. Such a test aims at verifying the effectiveness of the proposed strategy in a fault risk scenario, where one modality of the test sequence can be totally off-line. Figure 9 shows the CCC values of arousal (upper), and valence (lower) predictions, when the dynamic configuration is compared to the static solution. Note that, in this case, especially when the audio modality is totally missing, prediction performance drastically degrades, demonstrating once more the crucial role of the audio modality in emotion recognition, especially for arousal.

As a further important aspect, the proposed system has been specifically developed for real time applications when, after an initial training phase, the system is able to predict one sample at a time, in less than $400 \mathrm{~ms}$ that is the sampling time chosen in this study. All the techniques implemented in the approach have been selected since they require very small computational effort and can take advantage of matrix calculus (PLS, Euclidean distance among vectors, MRMS, etc.). Simulations were performed using Matlab 2017 ${ }^{\circledR}$, on an Intel Core i7 machine. Of course, for longer prediction session, a restricted number of training samples have to be chosen by definitely eliminating oldest training samples hence avoiding to calculate Euclidean distance over an even larger training set.

To conclude, it is important to highlight the potential application of the proposed strategy to diversified scenarios, such as chemical sensors, or signal processing in general. The approach presents an architecture that can be easily adapted to alternative frameworks with the aim to protect systems from failure and outof-range predictions. Moreover, even under the general assumption that there is not any superior configuration for sensor fusion, the C3 paradigm assumption is a fundamental step toward better performance in a large plethora of scenarios.

\section{Conclusion}

Despite the increasing interest, human emotion remains a complex, dynamically changing scenario that expresses itself in diversified human spheres. Therefore, a smart sensor network architecture is required for reliable prediction. In this paper, we presented a novel dynamic sensor network configuration that receives the emotion events communicated through the speech, the facial mimic, and physiological signals of a subject acquired while he/she is involved in natural conversation in a controlled environment. The shown system is able to continuously predict the two dimensions of affect (arousal and valence) under normal and simulated critical working conditions as demonstrated by specific experiments run. Although adequate performance was obtained in the experimental tests, more investigations are required in order to improve robustness, especially with the prediction of the emotional valence, that still appears as the most critical affective dimension. This is crucial especially in those applications where positive, neutral, and negative valence levels are used to calibrate the results of behavioural disorder studies conducted such as on children. To further verify the robustness of the prediction system, future work will also account for the presence of noise sources (wind noise, phone noise, car and train noises, etc.) investigating strategies for protecting the system from both soft and hard failures in real-life operation modes. 
464 The research leading to these results has been partially funded by the PainTCare project (University of Rome 465 Tor Vergata, Uncovering Excellence program), the European Commission's Seventh Framework Programme 466 through the ERC Starting Grant No. 338164 (iHEARu), and the European Union's Horizon 2020 Programme 467 through the Innovative Action No. 645094 (SEWA) and the Research Innovative Action No. 645378 (ARIA468 VALUSPA). 
[1] A. Mencattini, E. Martinelli, F. Ringeval, B. Schuller, C. Di Natale, Continuous Estimation of Emotions in Speech by Dynamic Cooperative Speaker Models, IEEE Transactions on Affective Computing, pages 14,2016, doi: 10.1109/TAFFC.2016.2531664.

[2] E. Martinelli, A. Mencattini, E. Daprati, C. Di Natale, Strength Is in Numbers: Can Concordant Artificial Listeners Improve Prediction of Emotion from Speech?, PloS one, 11, 8, pp. e0161752, 2016.

[3] F. Weninger, M. Wöllmer, B. Schuller, Emotion Recognition in Naturalistic Speech and Language-A Survey. Emotion Recognition: A Pattern Analysis Approach 237-267, 2015.

[4] M. Mansoorizadeh and N. M. Charkari, Multimodal information fusion application to human emotion recognition from face and speech, Multimedia Tools and Applications 49,2, pp. 277-297, 2010.

[5] R. A. Calvo and S. D'Mello. Affect detection: An interdisciplinary review of models, methods, and their applications, Affective Computing, IEEE Transactions on, 1, 1, pp.18-37, 2010.

[6] N. Sebe, I. Cohen. T. Gevers, T.S. Huang, Multimodal approaches for emotion recognition: a survey. In Electronic Imaging, pp. 56-67, International Society for Optics and Photonics 2005.

[7] J. Posner, J. Russell, and B. Peterson, "The circumplex model of affect: an integrative approach to affective neuroscience, cognitive development, and psychopathology," Develop. Psychopathol., 17, 3, pp. 715-734, 2005.

[8] Z. Zhang, F. Ringeval, B. Dong, E. Coutinho, E. Marchi, Enhanced semi-supervised learning for multimodal emotion recognition. In 2016 IEEE International Conference on Acoustics, Speech and Signal Processing (ICASSP) IEEE, pp. 5185-5189.

[9] F. Ringeval et al. AV+ EC 2015: The First Affect Recognition Challenge Bridging Across Audio, Video, and Physiological Data. In Proceedings of the 5th International Workshop on Audio/Visual Emotion Challenge, ACM, pp. 3-8, 2015.

[10] M. Valstar, J. Gratch, B. Schuller, F. Ringeval, D. Lalanne, M. T. Torres, S. Scherer, G. Stratou, R. Cowie, and M. Pantic. AVEC 2016 - Depression, Mood, and Emotion Recognition Workshop and Challenge. Proceedings of the 6th International Workshop on Audio/Visual Emotion Challenge, AVEC'16, colocated with the 24th ACM International Conference on Multimedia, MM 2016, pages 3-10, Amsterdam, The Netherlands, October 2016.

[11] A. Mencattini, F. Ringeval, B. Schuller, E. Martinelli, C. Di Natale, Continuous Monitoring of Emotions by a Multimodal Cooperative Sensor System. Procedia Engineering, 120, pp. 556-559, 2015.

[12]K.M. Tsang, W.L. Chan, Data validation of intelligent sensor using predictive filters and fuzzy logic, Sensors and Actuators A: Physical, 159, 2, pp. 149-156, 2010.

[13]W. Elmenreich, An introduction to sensor fusion. Vienna University of Technology, Austria (2002).

[14] E. Martinelli, G. Magna, S. De Vito, R. Di Fuccio, G. Di Francia, A. Vergara, C. Di Natale, An adaptive classification model based on the Artificial Immune System for chemical sensor drift mitigation. Sensors and Actuators B: Chemical 177, pp. 1017-1026, 2013.

[15] E. Martinelli, G. Magna, A. Vergara, C. Di Natale, Cooperative classifiers for reconfigurable sensor arrays. Sensors and Actuators B: Chemical, 199, pp. 83-92, 2014.

[16] G. Magna, F. Mosciano, E. Martinelli, C. Di Natale, An on-line reconfigurable classification algorithm improves the long-term stability of gas sensor arrays in case of faulty and drifting sensors. Procedia Engineering, 120, pp. 249-252, 2015.

[17] J. Yao, H. Zhang, X. Xiang, H. Bai, Y. Zhao, A 3-D printed redundant six-component force sensor with eight parallel limbs. Sensors and Actuators A: Physical, 247, pp. 90-97, 2016.

[18]L. Fernandez, S. Marco, A. Gutierrez-Galvez, Robustness to sensor damage of a highly redundant gas sensor array, Sensors and Actuators, B: Chemical, 218, pp. 296-302. 
[19]J. Fonollosa, A. Vergara, R. Huerta, Algorithmic mitigation of sensor failure: Is sensor replacement really necessary? Sensors and Actuators, B: Chemical, 183, pp. 211-221, 2013.

[20] W.-T. Sung, and K.-Y. Chang, Evidence-based multi-sensor information fusion for remote health care systems, Sensors and Actuators A: Physical, 204, pp. 1-19, 2013.

[21] F. Ringeval, A. Sonderegger, J. Sauer, D. Lalanne, Introducing the RECOLA multimodal corpus of remote collaborative and affective interactions. Automatic Face and Gesture Recognition (FG), 2013 10th IEEE International Conference and Workshops on. IEEE, pp. 1-8, 2013.

[22] M. Grimm, K. Kroschel, S. Narayanan, The Vera am Mittag German audio-visual emotional speech database, in: Proc. of the 8th Inter. Conf. on Multimedia \& Expo (ICME), Hannover, Germany. pp. 865868, 2008.

[23] W.J. Yan, Q. Wu, J. Liang, Y.H. Chen, W. Fu, How fast are the leaked facial expressions: The duration of micro-expressions. Journal of Nonverbal Behavior 37, pp. 217-230, 2013.

[24] B. Schuller, S. Steidl, A. Batliner, A. Vinciarelli, K. Scherer, F. Ringeval, M. Chetouani, F. Weninger, F. Eyben, E. Marchi, M. Mortillaro, H. Salamin, A. Polychroniou, F. Valente, and S. Kim. The INTERSPEECH 2013 Computational Paralinguistics Challenge: Social Signals, Conflict, Emotion, Autism. In Proceedings INTERSPEECH 2013, 14th Annual Conference of the International Speech Communication Association, pages 148-152, Lyon, France, August 2013. ISCAX. Xiong and F. De la Torre. Supervised descent method and its applications to face alignment. In Proc. of CVPR, pp. 532539, Portland (OR), USA, 2013. IEEE.

[25] P. Ekman, W.V. Friesen, Facial action coding system: A technique for the measurement of facial movement. Palo Alto, CA: Consulting Psychologists Press, 1978.

[26] D. Lowe, Distinctive image features from scale-invariant keypoints, Intern. Journal of Computer Vision, 60, pp. 91-110, 2004.

[27] J. Xiao, T. Moriyama, T. Kanade, J.F. Cohn, Robust full-motion recovery of head by dynamic templates and re-registration techniques. Inter. Journal of Imaging Systems and Technology, 13, pp. 85-94, 2003.

[28]G. Farneback, Two-frame motion estimation based on polynomial expansion, in: Image Analysis, Special Issue, 13th Scandinavian Conf. (SCIA). Springer, Halmstad, Sweden. volume 2749, pp. 363370, 2003.

[29]F. Ringeval, F. Eyben, E. Kroupi, A. Yuce, J.P. Thiran, T. Ebrahimi, D. Lalanne, B.Schuller Prediction of asynchronous dimensional emotion ratings from audiovisual and physiological data. Pattern Recognition Letters, 66, pp. 22-30, 2015.

[30] M. Dawson, A. Schell, D. Filion, The electrodermal system, in: Cacioppo, J.T., Tassinary, L.G., Berntson, G.G. (Eds.), Handbook of psychophysiology. Cambridge: Cambridge University Press. volume 2, pp. 200-223, 2007.

[31] R. B. Knapp, J. Kim, and E. André. Physiological signals and their use in augmenting emotion recognition for human-machine interaction. In Emotion-Oriented Systems - The Humaine Handbook, pp. 133-159. Springer Berlin Heielberg, 2011.

[32] S. Koelstra, C. Mühl, M. Soleymani, J.-S. Lee, A. Yazdani, T. Ebrahimi, T. Pun, and A. N. I. Patras. DEAP: A database for emotion analysis using physiological signals. IEEE Transactions on Affective Computing, 3, pp. 18-31, 2012.

[33] S. Schachter. Cognition and peripheralist-centralist controversies in motivation and emotion. In M. S. Gazzaniga, editor, Handbook of Psychobioogy, pp. 529-564. Academic Press Inc., 2012.

[34] D. Keltner and J. S. Lerner. Emotion. In S. Fiske, D. Gilbert, and G. Lindzey, editors, Handbook of Social Psychology, 1, pp. 317-331. John Wiley \& Sons Inc., $5^{\text {th }}$ edition, 2010. 
[35] A. Sanoa, R. W. Picard, and R. Stickgold. Quantitative analysis of wrist electrodermal activity during sleep, International Journal of Psychophysiology, 94, 3, pp. 382-389, 2014.

[36] R. Picard. Affective media and wearables: surprising findings. In Proc. of ACM MM, pp. 3-4, Orlando (FL), USA, 2014. ACM.

[37] M. Chen, Y. Zhang, M. M. H. Yong Li, and A. Alarmi. AIWAC: Affective interaction through wearable computing and cloud technology, IEEE Mobile Wearable Communications, 22, 1, pp. 20-27, 2015.

[38] H. Peng, F. Long, C. Ding, Feature selection based on mutual information criteria of max-dependency, max-relevance, and min-redundancy, IEEE Trans. Pattern Anal. Mach. Intell. 27 (8) (2005) 1226-1238.

[39]S. Wold, M. Sjöström, L. Eriksson, PLS-regression: a basic tool of chemometrics, Chemometr. Intell. Lab. Syst. 58 (2) (2001) 109-130.

[40]V. Vinzi, L. Trinchera, S. Amato, PLS path modeling: from foundations to recent developments and open issues for model assessment and improvement, in: Handbook of Partial Least Squares, Springer, 2010, pp. 47-82.

[41]X.-S. Gan, J.-S. Duanmu, J.-F. Wang, W. Cong, Anomaly intrusion detection based on PLS feature extraction and core vector machine, Knowl.-Based Syst. 40 (2013) 1-6.

[42]S.-S. Chen, Y.-W. Chuang, P.-Y. Chen, Behavioral intention formation in knowledge sharing: examining the roles of KMS quality, KMS self-efficacy, and organizational climate, Knowl.-Based Syst. 31 (2012) 106-118.

[43] S. de Jong, SIMPLS: an alternative approach to partial least squares regression, Chemometr. Intell. Lab. Syst. 18 (3) (1993) 251-263.

[44]I.-K. L. Lin, A concordance correlation coefficient to evaluate reproducibility, Biometrics, 45, 1, pp. 255-268, 1989.

[45] F. Weninger, F. Ringeval, E. Marchi, and B. Schuller. Discriminatively Trained Recurrent Neural Networks for Continuous Dimensional Emotion Recognition from Audio. In Proceedings of the 25th International Joint Conference on Artificial Intelligence (IJCAI 2016), pp. 2196-2202, New York City (NY), USA, July 2016. IJCAI/AAAI. 
598 Tables Captions:

599 Table 1. Metadata of the 10 speakers used in this work.

600 Table 2. CCC median and median absolute values for the arousal and valence prediction using the dynamic 601 single modality configuration: multi (multimodal).

602

603

604

605

606

607 
Table 1.

\begin{tabular}{|cccccc|} 
Speaker Label & Age & Sex & Mother tongue & Arousal $(\mu \pm \sigma)$ & Valence $(\mu \pm \sigma)$ \\
\hline P16 & 21 & M & French & $0.03 \pm 0.17$ & $0.13 \pm 0.10$ \\
P19 & 20 & $F$ & French & $0.09 \pm 0.15$ & $0.12 \pm 0.13$ \\
P21 & 19 & F & Italian & $0.03 \pm 0.15$ & $0.11 \pm 0.11$ \\
P28 & 18 & F & French & $-0.09 \pm 0.14$ & $0.09 \pm 0.12$ \\
P30 & 22 & F & Italian & $-0.15 \pm 0.19$ & $0.03 \pm 0.14$ \\
P34 & 25 & M & French & $-0.19 \pm 0.17$ & $-0.02 \pm 0.05$ \\
P41 & 23 & M & German & $0.15 \pm 0.10$ & $0.11 \pm 0.09$ \\
P45 & 19 & F & French & $-0.02 \pm 0.19$ & $0.08 \pm 0.09$ \\
P56 & 22 & M & French & $0.07 \pm 0.16$ & $0.12 \pm 0.11$ \\
P64 & 25 & M & French & $0.01 \pm 0.14$ & $0.08 \pm 0.07$
\end{tabular}

609 
Table 2.

\begin{tabular}{|c|c|c|c|c|c|c|c|c|c|c|}
\hline \multicolumn{6}{|c|}{ Arousal } & \multicolumn{5}{|c|}{ Valence } \\
\hline & multi & audio & video & ecg & eda & multi & audio & Video & ecg & eda \\
\hline median CCC & 0.622 & 0.587 & 0.117 & 0.098 & -0.005 & 0.303 & 0.093 & -0.022 & 0.028 & 0.050 \\
\hline std. dev. & 0.121 & 0.111 & 0.132 & 0.119 & 0.063 & 0.266 & 0.166 & 0.259 & 0.092 & 0.145 \\
\hline
\end{tabular}

612

613

614

615

616

617

618

619

620

621

622

623

624

625

626

627

628

629

630

631

632

633

634

635

636 
Figure 1. Graphical representation of the problems to account for in a personalized multimodal mood estimation framework.

Figure 2. Distribution over the 10 speakers (denoted with P16 - P64) of the average absolute correlation coefficient (CC) of the features in each modality (distinguished by colours) with respect to the arousal (top) and valence dimension 642 (bottom).

Figure 3. A schematic illustration of the proposed method. The missing measure protection (MMP) module identifies features/sensor modality that are spurious or missing in the test input and eliminate them from the training set. The dynamic training template recruitment (DTTR) module extracts from the reduced training set the templates most affine to the reduced test input and sends them to the feature selection module based on maximum relevance modality selection (MRMS) criterion. Selected training templates are then used to construct the regression module (RM) that assigns a predicted output to the test input (test output). Finally, the dynamic measure integration (DMI) module aims at including the predicted output into the training set to enlarge the knowledge-base.

Figure 4. Boxplot of the concordance correlation coefficients (CCC) computed for the dynamic multimodal configuration of the arousal (first column) and valence (third column) prediction against the static multimodal configuration for arousal (second column) and valence (fourth column) (Test 1). The p-values of the improvement quantified by running paired t-test on the CCC values are also reported. Dyn Mult Arousal and Dyn Mult Valence indicate the proposed dynamic multimodal prediction of arousal and valence, respectively. Stat Mult Arousal and Stat Mult Valence indicate the standard static multimodal prediction of arousal and valence, respectively.

Figure 5. Two examples of arousal (top) and valence (bottom) prediction for subject P16. The blue line indicates the achieved prediction, the red line represents the expected output, and the green squares locate the training session. The corresponding CCC values are also reported.

Figure 6. Boxplot of the concordance correlation coefficients (CCC) computed for dynamic multimodal configuration of the arousal (upper) and valence (lower) prediction against the static multimodal configuration in case of $10 \%$ of features for each modality in turn randomly set to missing or spurious values (Test 2). Dyn Mult Arousal and Dyn Mult Valence indicate the proposed dynamic multimodal prediction of arousal and valence, respectively. Stat Mult Arousal and Stat Mult Valence indicate the standard static multimodal prediction of arousal and valence, respectively.

Figure 7. Relative frequency of selection of features of each modality during prediction of arousal (left) and valence (right) in the dynamic configuration.

Figure 8. Progressive spurious feature injection during valence prediction in the video modality. Three time instants are shown (80s, 160s, 268s). Bar colours indicate the four modalities as explained in the legend. Bottom bars represent the percentage of non-spurious features for each modality. Top bars indicate the percentage of selected features for each modality.

Figure 9. Boxplot of the concordance correlation coefficients (CCC) computed for dynamic multimodal configuration of the arousal (upper) and valence (lower) prediction against the static multimodal configuration in case of modality missing in turn. Dyn Mult Arousal and Dyn Mult Valence indicate the proposed dynamic multimodal prediction of arousal and valence, respectively. Stat Mult Arousal and Stat Mult Valence indicate the standard static multimodal prediction of arousal and valence, respectively. 


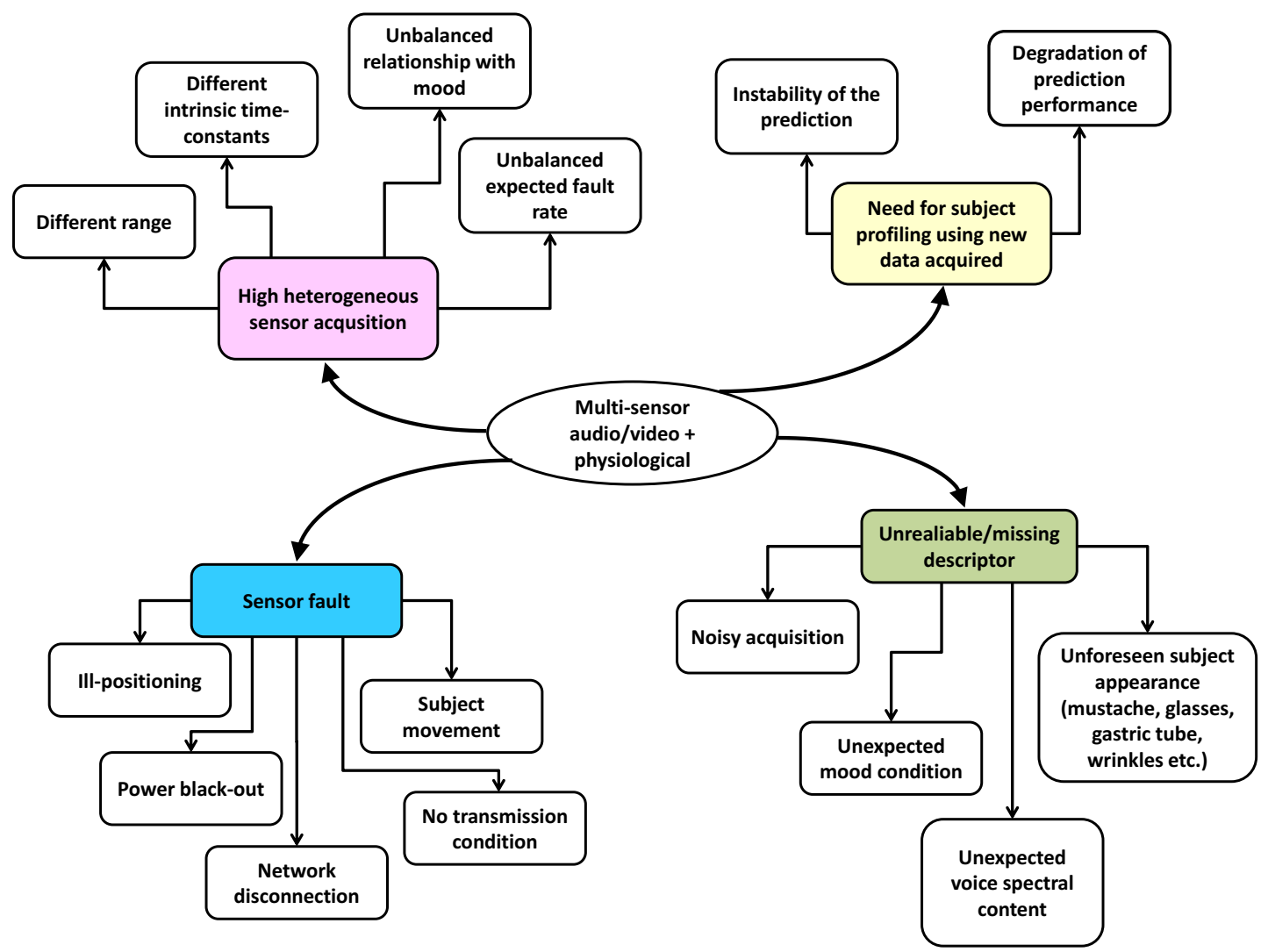

$676 \quad$ Figure 1. 

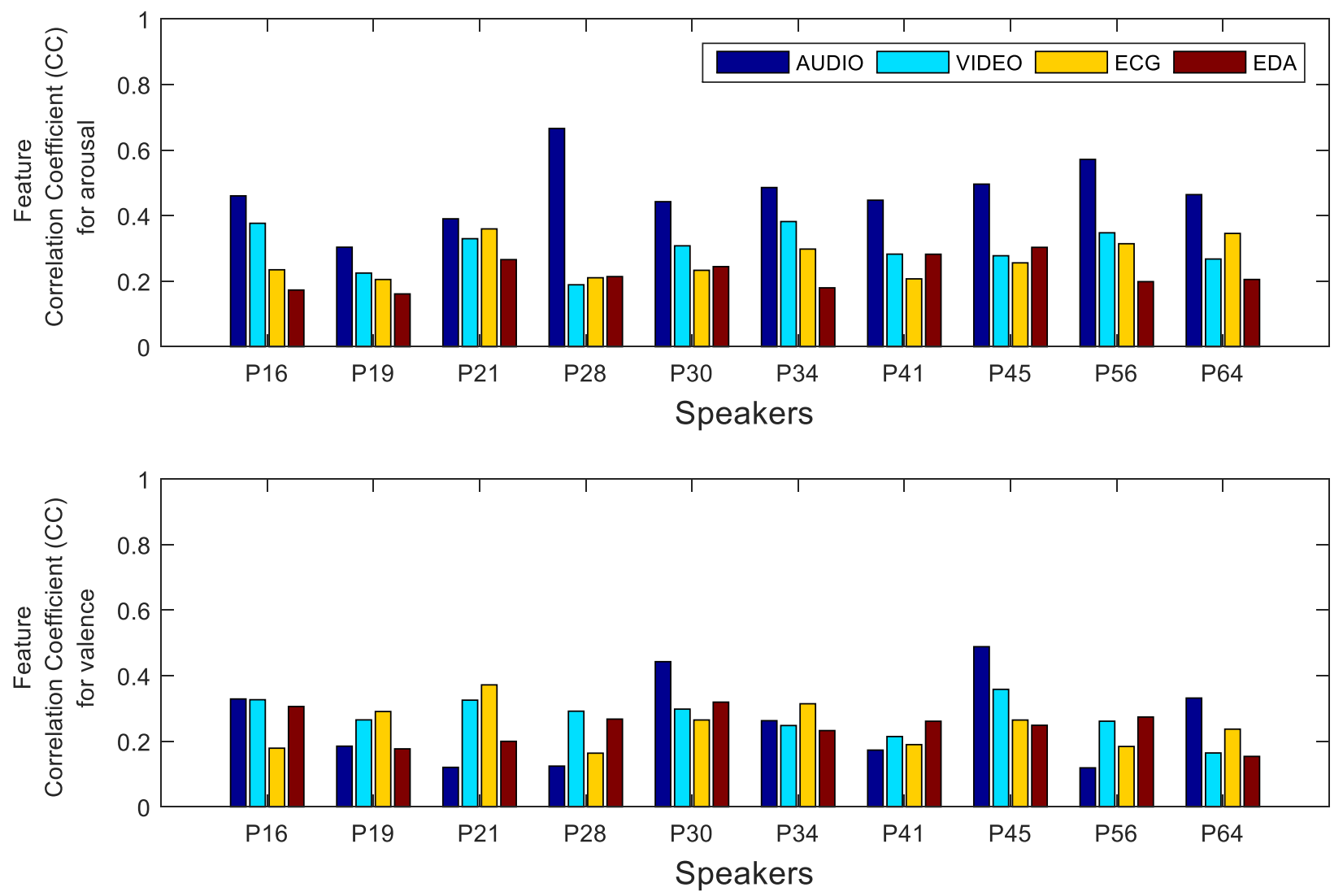

678

$679 \quad$ Figure 2.

680 
Incoming unknown affective condition

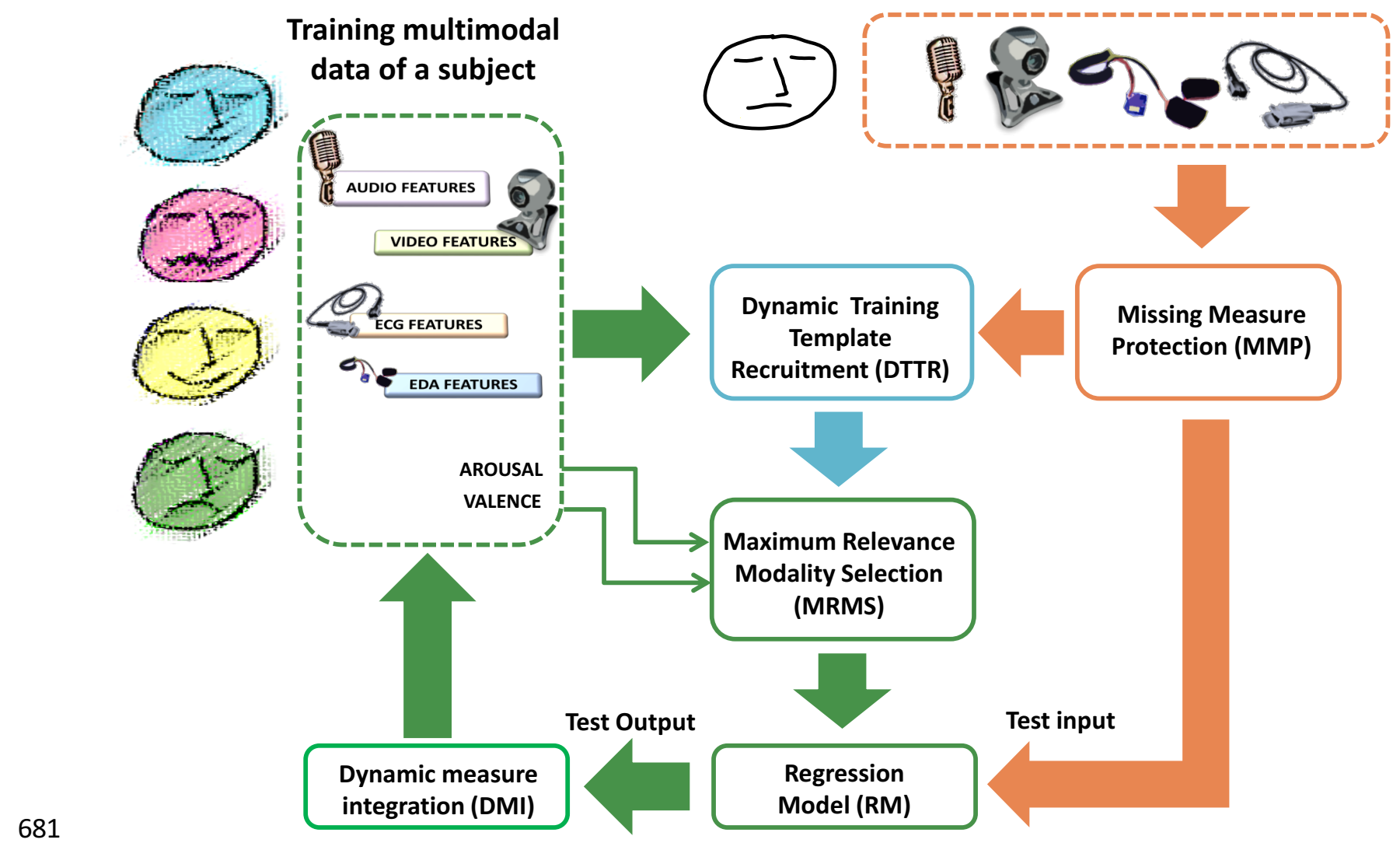

Figure 3. 


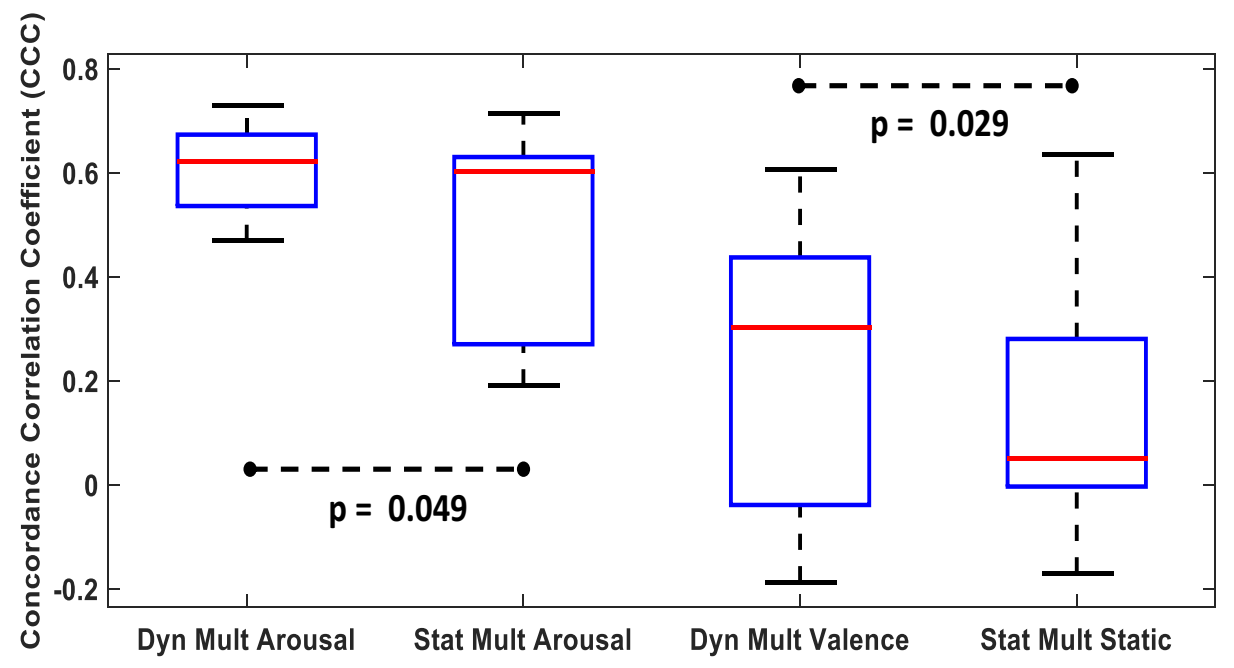

685

$686 \quad$ Figure 4.

687 

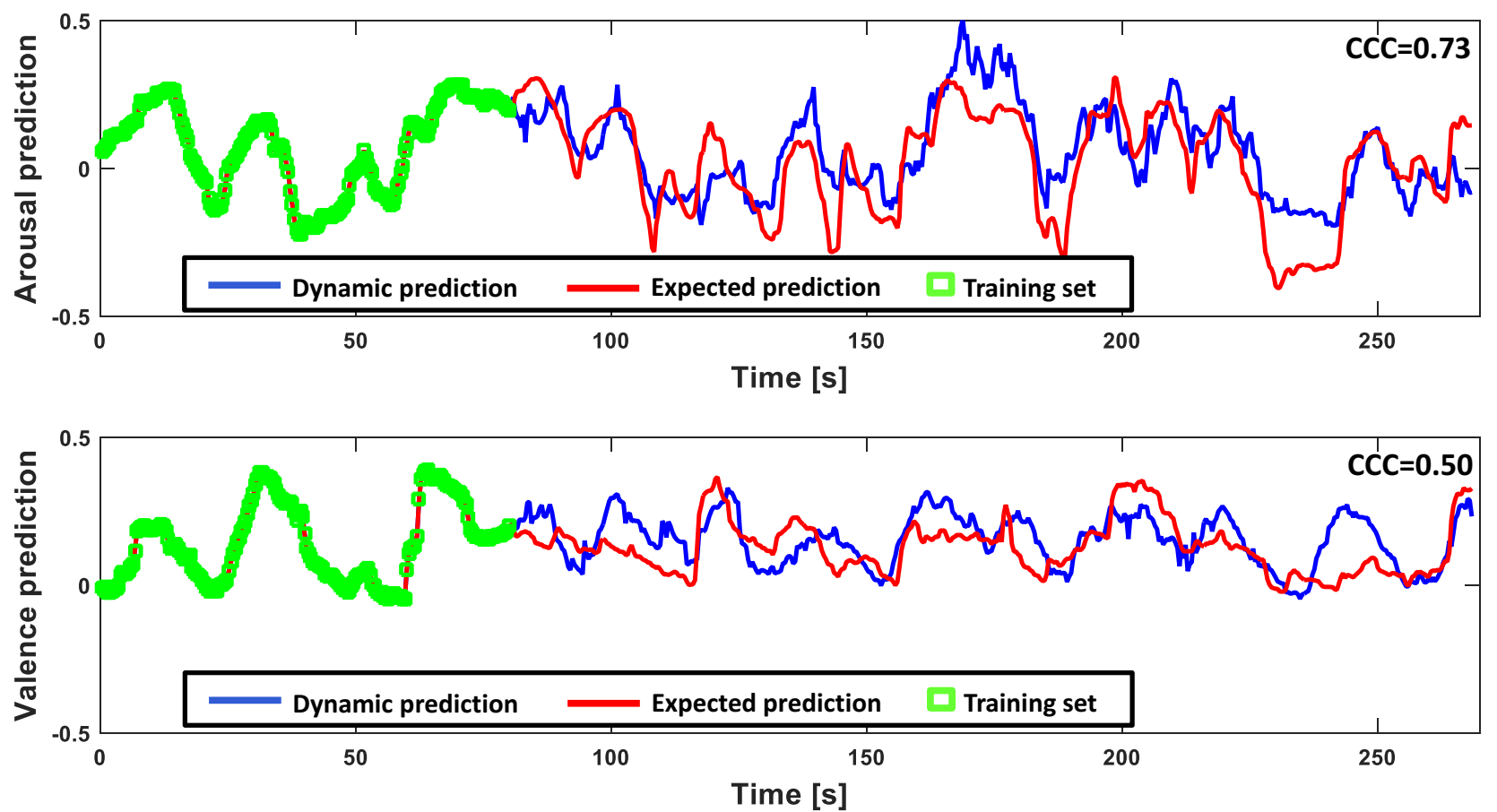

688

$689 \quad$ Figure 5.

690 
694

695

696

697

698

699

700
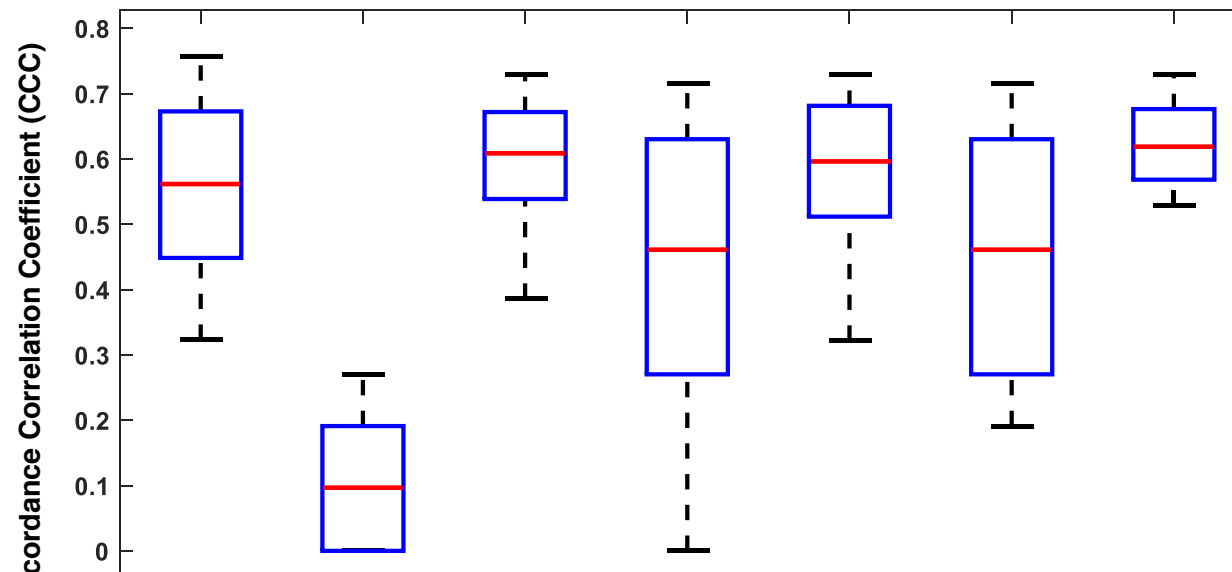
arousal

701

702

703

704

705

706

707

708

709

710

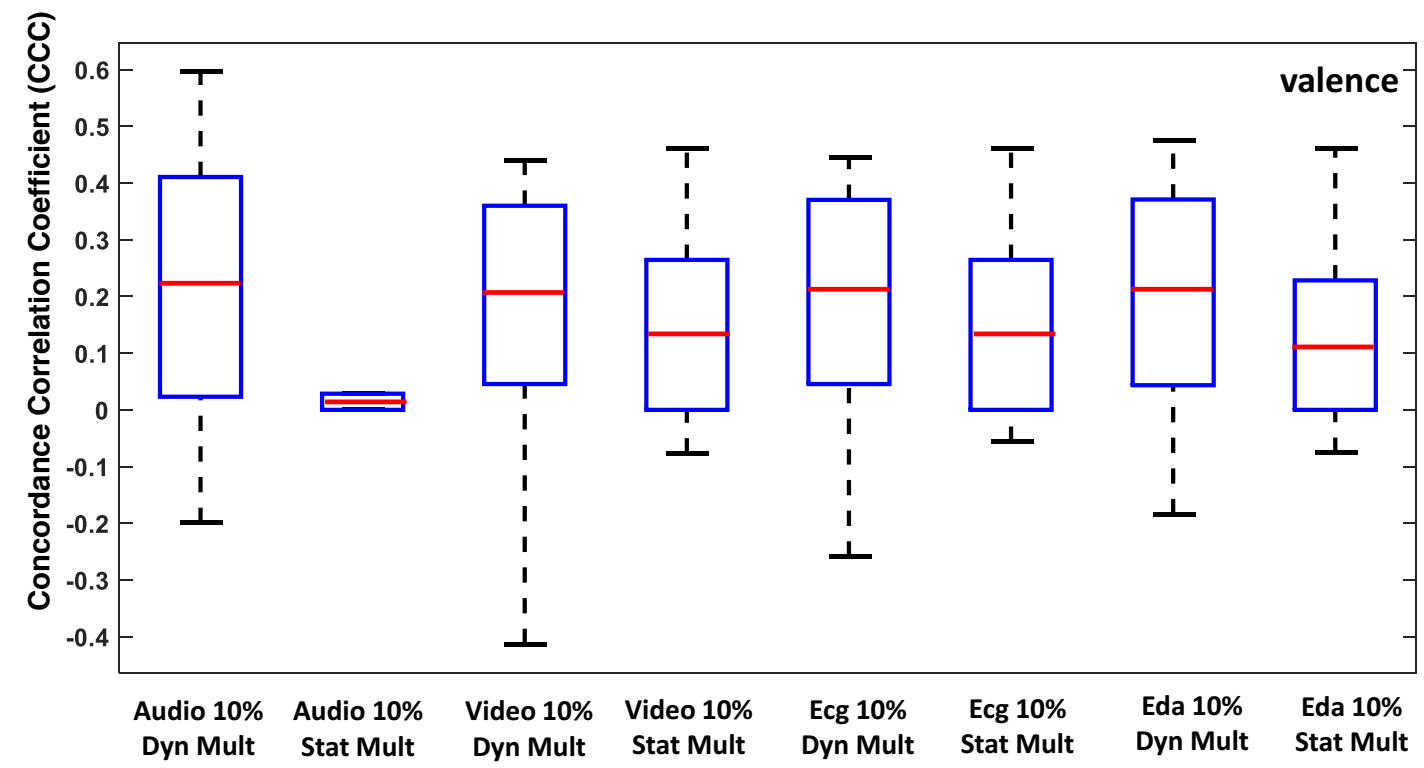

711

712

Figure 6.

713

714

715 

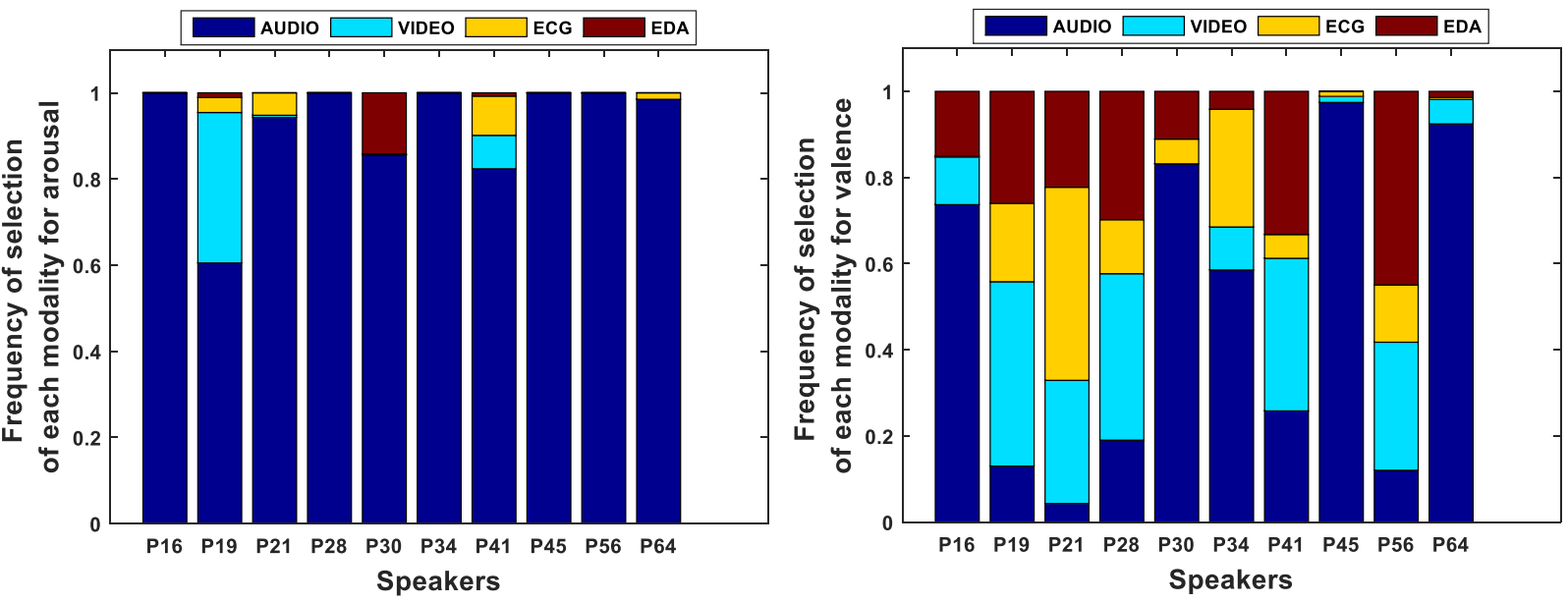

718

Figure 7.

719

720

721

722

723

724

725

726

727

728

729 


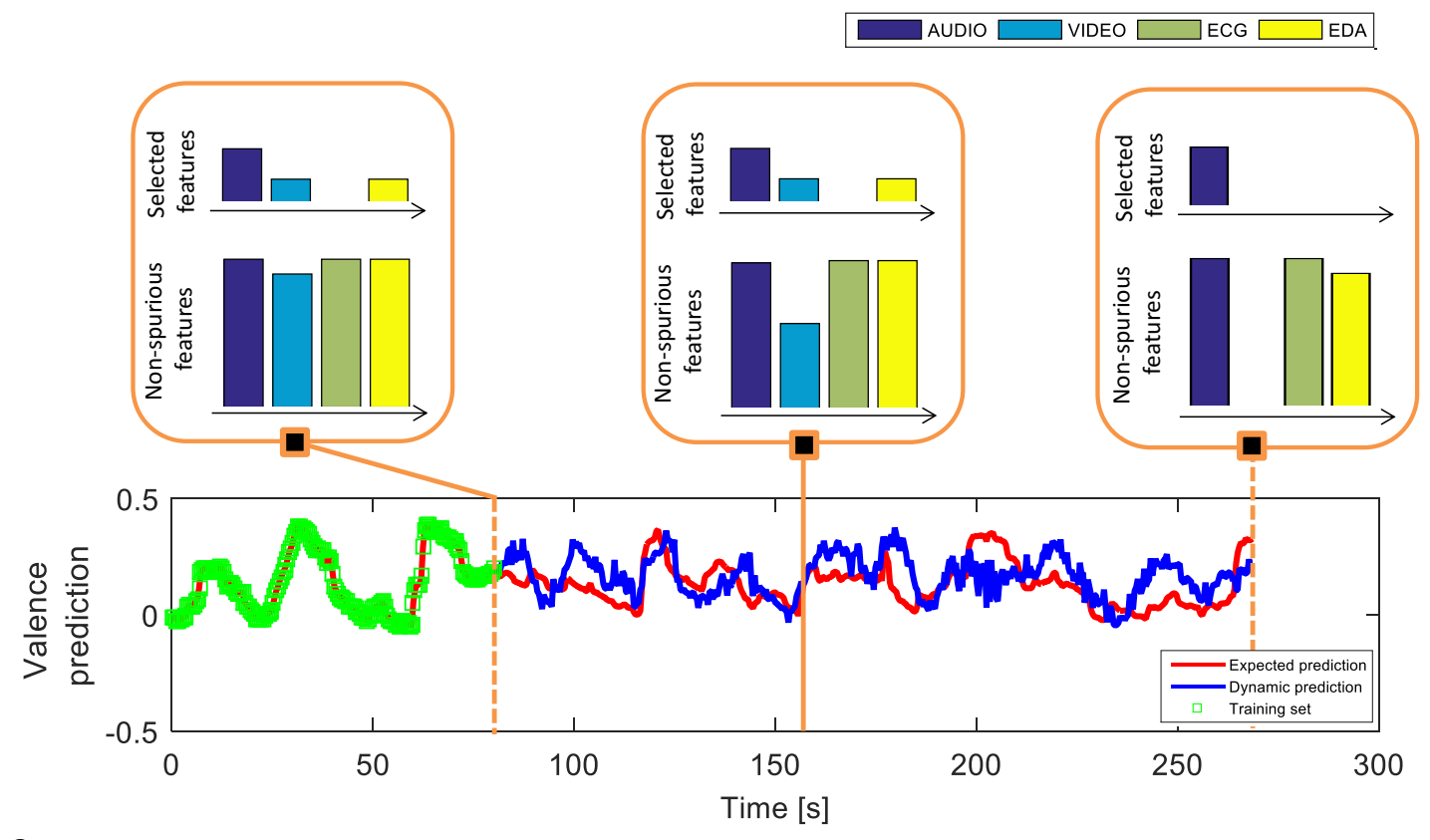

$730 \quad$ Figure 8.

731

732

733

734

735

736

737

738

739

740

741

742

743

744

745

746

747

748 


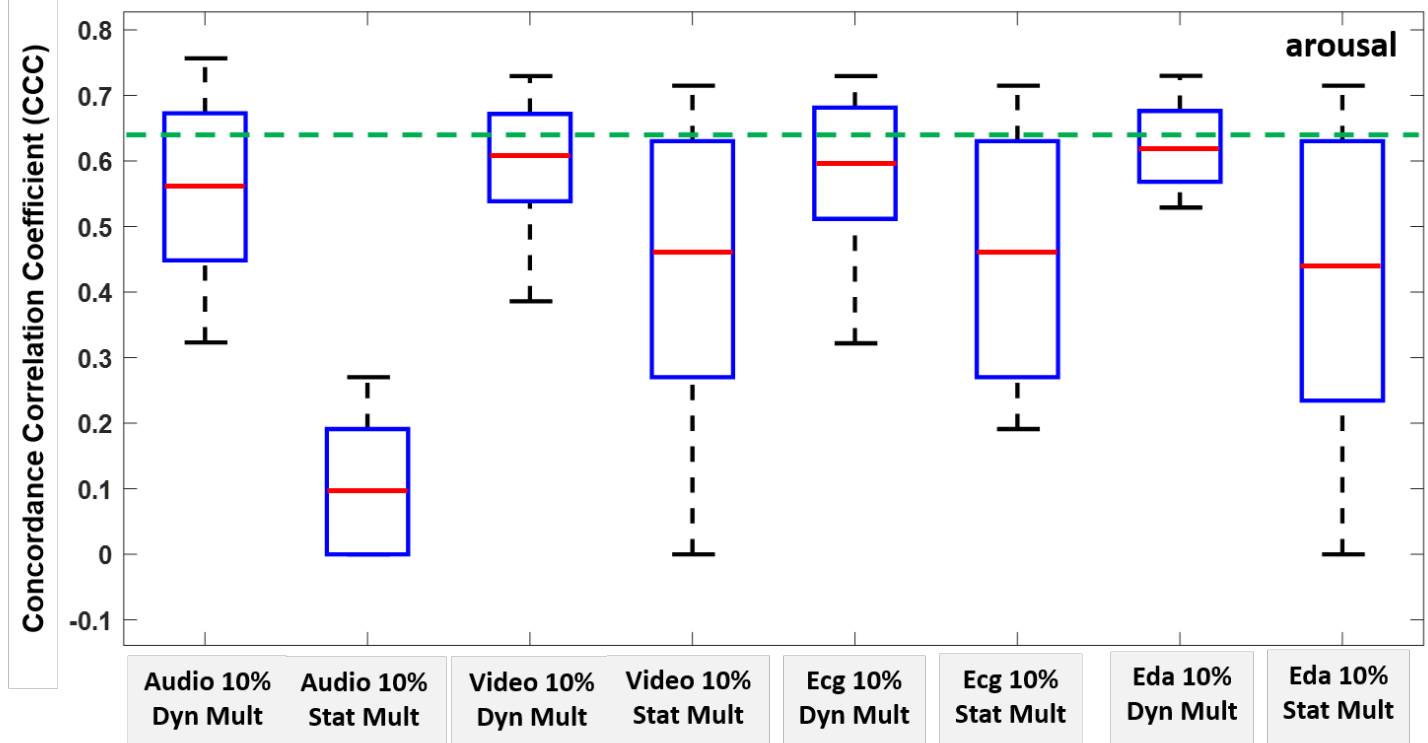

751

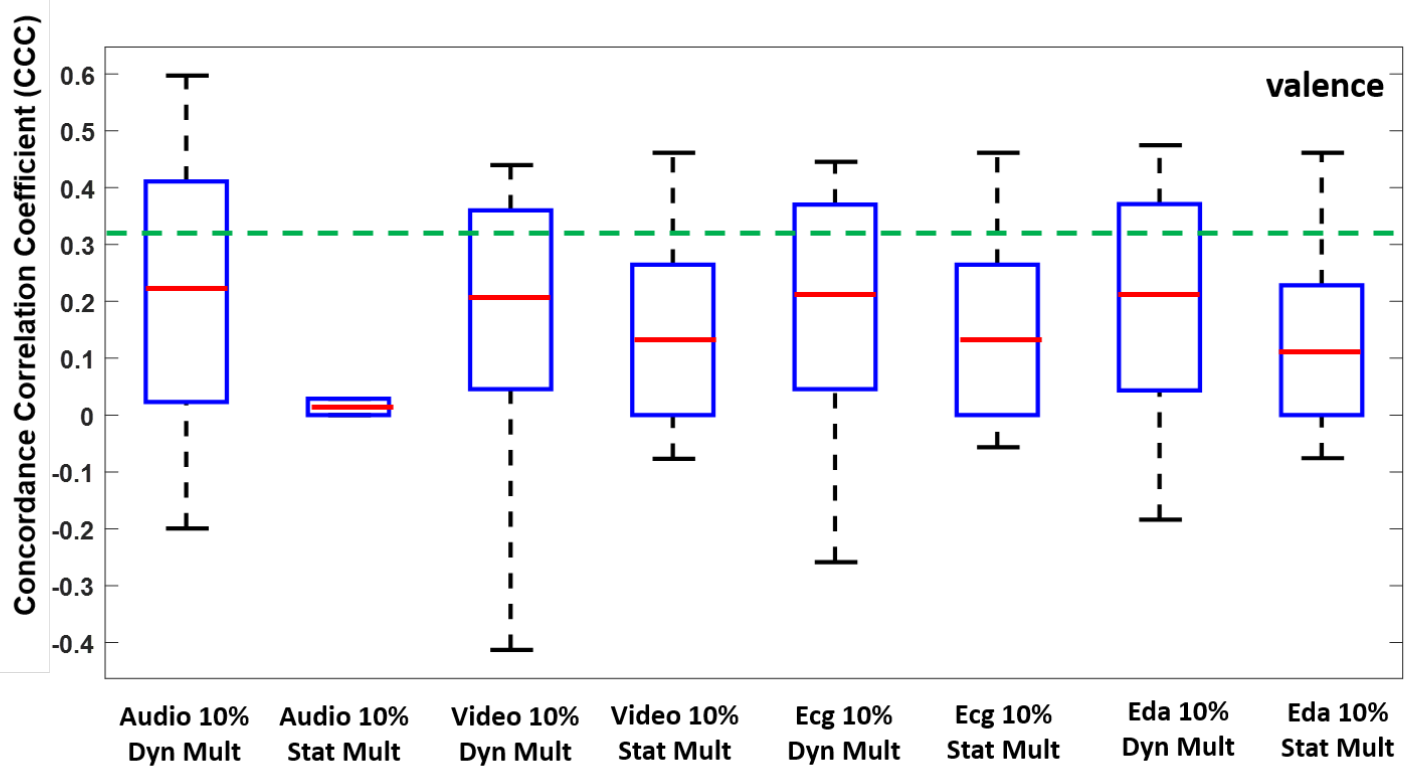

Figure 9. 